\title{
THE TOPOLOGICAL COMPLEXITY OF THE FREE PRODUCT
}

\author{
ALEXANDER DRANISHNIKOV, RUSTAM SADYKOV
}

\begin{abstract}
We prove the formula
$\mathrm{TC}(G * H)=\max \{\mathrm{TC}(G), \mathrm{TC}(H), c d(G \times H)\}$

for the topological complexity of the free product of groups with cohomological dimension $\geq 3$.
\end{abstract}

\section{INTRODUCTION}

Let $X$ be a topological space. The Lusternik-Schnirelmann category (LS-category) cat $X$ of $X$ is the least number $n$ such that there is a covering $\left\{U_{i}\right\}$ of $X$ by $n+1$ open sets $U_{i}$ contractible in $X$ to a point. The LS-category has a number of interesting applications [5]. A motion planning algorithm over an open subset $U_{i} \subset X \times X$ is a continuous map $U_{i} \rightarrow X^{[0,1]}$ that takes a pair $(x, y)$ to a path $s$ with end points $s(0)=x$ and $s(1)=y$. The topological complexity $\operatorname{TC}(X)$ of $X$ is the least number $n$ such that there is a covering $\left\{U_{i}\right\}$ of $X \times X$ by $n+1$ open sets over which there are motion planning algorithms [11]. We note that in this paper we consider the reduced LS-category and the reduced topological complexity.

Both cat $X$ and TC $(X)$ are homotopy invariant. Thus one can define the LScategory and the topological complexity of a discrete group $G$ by setting cat $G=$ cat $B G$ and $\mathrm{TC}(G)=\mathrm{TC}(B G)$ where $B G=K(G, 1)$ is a classifying spaces for $G$. It is well-known that cat $G$ does not give a new invariant [10, [18], [19].

Theorem 1 (Eilenberg-Ganea). For all groups cat $G=c d(G)$.

Here $\operatorname{cd}(G)$ is the cohomological dimension of $G$. By the Eilenberg-Ganea theorem $c d(G)$ equals the geometric dimension $\operatorname{gdim}(G)=\min \{\operatorname{dim} B G\}$ of $G$ provided $c d(G) \neq 2$ [2]. The Eilenberg-Ganea conjecture states that the equality $c d(G)=$ $\operatorname{gdim}(G)$ holds for all groups. A potential counterexample to Eilenberg-Ganea conjecture should have $\operatorname{cd}(G)=2$ and $\operatorname{gdim}(G)=32$.

The topological complexity of a group is secluded in the range $c d(G) \leq \mathrm{TC}(G) \leq$ $2 c d(G)$ and any value between can be taken [16]. Computation of topological complexity of a group is a great challenge. Since TC $(G)=\infty$ for groups with torsion, this invariant makes sense only for torsion free groups. It was computed only for few classes of groups. Thus, the topological complexity of free abelian group equals the rank. The topological complexity of nilpotent groups is estimated in [14]. Computation of topological complexity of surface groups orientable and non-orientable was only recently completed [11, [8], [4]. Even more recent is the computation of TC of hyperbolic groups [13].

In this paper we present a formula for topological complexity of free product of groups. 
Theorem 2. If both groups $G$ and $H$ are not counterexamples to the Eilenberg-Ganea conjecture, then

$$
\mathrm{TC}(G * H)=\max \{\mathrm{TC}(G), \mathrm{TC}(H), c d(G \times H)\} .
$$

In view of the inequality $\mathrm{TC}(X) \leq \operatorname{cat}(X \times X)$ we obtain the following:

Corollary 3. If $G$ is not a counterexample to the Eilenberg-Ganea conjecture, then

$$
\mathrm{TC}(G * G)=c d(G \times G) .
$$

Corollary 4.

$$
\mathrm{TC}\left(\mathbb{Z}^{m} * \mathbb{Z}^{n}\right)=m+n .
$$

Corollary 5 (Yu. Rudyak). For any $n$ and $k$ with $n \leq k \leq 2 n$ there is a group $G$ having $\operatorname{cd}(G)=n$ and $\mathrm{TC}(G)=k$

Proof. Take $G=\mathbb{Z}^{m} * \mathbb{Z}^{n}$ for $m=k-n$.

REMARK 1. If $G$ (or $H$ ) is a counterexample to the Eilenberg-Ganea conjecture having $\mathrm{TC}(G)=4(\mathrm{TC}(H)=4)$, then still Theorem 2 holds true.

REMARK 2. Theorem 2 holds for groups $G$ and $H$ with $c d(G) \geq c d(H) \geq 2$ and $c d(G \times H) \geq c d(G)+2$. We beleive that the latter inequality holds true for all geometrically finite groups $H$ with $c d(H) \geq 2$. We note that raising dimension by 2 of the Cartesian product with a nonfree group is the maximal possible in view of an example in [7] of geometrically finite groups $G$ and $H$ with $c d(G)=c d(H)=3$ and $c d(G \times H) \leq 5$. The groups $G$ and $H$ in [7] are finite index subgroups of right-angled Coxeter groups constructed by an appropriate choice of the nerves. Also we note that in the class of infinitely generated groups there is a counterexample: $c d(\mathbb{Q})=2$ while $c d(\mathbb{Q} \times \mathbb{Q})=3$. We are thankful to David Recio-Mitter for the latter remark.

\section{Topological COMplexity of WedGe}

It was proved in [6], Theorem 3.6, that

$$
\max \{\mathrm{TC} X, \mathrm{TC} Y, \operatorname{cat}(X \times Y)\} \leq \mathrm{TC}(X \vee Y) \text {. }
$$

We show that under certain conditions the lower estimate is exact.

Theorem 6. Let $d=\max \{\operatorname{dim} X, \operatorname{dim} Y\}$ for connected $C W$-complexes $X$ and $Y$. Suppose that $\max \{\mathrm{TC} X, \mathrm{TC} Y$, cat $(X \times Y\} \geq d+1$. Then

$$
\mathrm{TC}(X \vee Y)=\max \{\mathrm{TC} X, \mathrm{TC} Y, \operatorname{cat}(X \times Y)\} \text {. }
$$

We note that in many cases this is an improvement of the upper bound from [6]

$$
\mathrm{TC}(X \vee Y) \leq \mathrm{TC}^{M} X+\mathrm{TC}^{M} Y
$$

where TC ${ }^{M}$ is the monoidal topological complexity (see [15]). We recall that TC $X \leq$ $\mathrm{TC}^{M} \leq \mathrm{TC} X+1$. In view of the fact that cat $(X \times Y) \leq \operatorname{cat} X+\operatorname{cat} Y$, Theorem 6 implies the inequality conjectured in [12], [6, Remark 3.7]

$$
\mathrm{TC}(X \vee Y) \leq \max \{\mathrm{TC} X, \mathrm{TC} Y, \operatorname{cat} X+\operatorname{cat} Y\}
$$

under the hypothesis of Theorem 6 .

We extend Theorem [6 in two directions. First observation is that the condition of Theorem [6 can be relaxed in case of $r$-connected complexes. 
Theorem 7. Let $d=\max \{\operatorname{dim} X, \operatorname{dim} Y\}$ for $r$-connected $C W$-complexes $X$ and $Y$. Suppose that $\max \left\{\mathrm{TC} X, \mathrm{TC} Y\right.$, cat $(X \times Y\} \geq \frac{d+1}{r+1}$. Then

$$
\mathrm{TC}(X \vee Y)=\max \{\mathrm{TC} X, \mathrm{TC} Y, \operatorname{cat}(X \times Y)\} \text {. }
$$

We note that the condition TC $X \geq(\operatorname{dim} X+1) /(r+1)$ for $r$-connected complexes has been seen before in the TC theory. Thus, the first author showed [6] that the topological complexity TC $X$ coincides with the monoidal topological complexity TC ${ }^{M} X$ for $r$-connected $X$ under the assumption that TC $X \geq(\operatorname{dim} X+1) /(r+1)$.

Then we extend the upper bound of Theorem 6 from the wedge of two spaces to the union.

Theorem 8. Let $d=\max \{\operatorname{dim}(X \times C), \operatorname{dim}(Y \times C)\}$ for connected $C W$-complexes $X$ and $Y$ where $C=X \cap Y$. Suppose that $\max \{\mathrm{TC} X$, TC $Y$, cat $(X \times Y\} \geq d+1$. Then

$$
\mathrm{TC}(X \cup Y) \leq \max \{\mathrm{TC} X, \mathrm{TC} Y, \operatorname{cat}(X \times Y)\}
$$

\section{Preliminaries}

3.1. Fiberwise join. The join $X_{0} * X_{1} * \cdots * X_{n}$ of topological spaces consists of formal linear combinations $t_{0} x_{0}+\cdots+t_{n} x_{n}$ of points $x_{i} \in X_{i}$ with non-negative coefficients $t_{i}$ that satisfy the condition $\sum t_{i}=1$. The fiberwise join of the total spaces $X_{0}, \ldots, X_{n}$ of fibrations $f_{i}: X_{i} \rightarrow Y$ is defined to be the topological space

$X_{0} *_{Y} X_{1} *_{Y} \cdots *_{Y} X_{n}=\left\{t_{0} x_{0}+\cdots+t_{n} x_{n} \in X_{0} * \cdots * X_{n} \mid f_{0}\left(x_{0}\right)=\cdots=f_{n}\left(x_{n}\right)\right\}$.

It is fibered over $Y$ by means of the map, called the fiberwise join of fibrations, defined by taking a point $t_{0} x_{0}+\cdots+t_{n} x_{n}$ to $f_{i}\left(x_{i}\right)$ for any $i$. As the name 'fiberwise join' suggests, the fiber of the fiberwise join of fibrations is given by the join of fibers of fibrations.

In all our applications, the spaces $X_{i}$ coincide with a space $X$ and all fibrations $f_{i}$ coincide with a fibration $f$ over a space $Y$. In this case the fiberwise join and the fiberwise join of fibrations are denoted by $*_{Y}^{n+1} X$ and $*_{Y}^{n+1} f$ respectively.

Let $G_{X}^{0}$ be the space of paths $s:[0,1] \rightarrow X$ issued from the base point of $X$. It is fibered over $X$ by means of the map $s \mapsto s(1)$. By definition, the $n$-th Ganea space of $X$ is the fiberwise join $G_{X}^{n}=*^{n+1} G_{X}^{0}$. By the Schwarz theorem [17], $\operatorname{cat}(X) \leq n$ if and only if the $n$-th Ganea fibration $p_{X}^{n}: G_{X}^{n} \rightarrow X$ admits a section.

Similarly, let $P X$ denote the space of paths $s:[0,1] \rightarrow X$. It is fibered over $X \times X$ by means of the map $s \mapsto(s(0), s(1))$. Let $\Delta_{X}^{n}$ denote the fiberwise join $*^{n+1} P X$. Then, by the Schwarz theorem, TC $X \leq n$ if and only if $\Delta_{X}^{n}$ admits a section.

3.2. The Berstein-Schwarz class. Let $\pi$ be a discrete group and $A$ be a $\pi$-module. By $H^{*}(\pi, A)$ we denote the cohomology of the group $\pi$ with coefficients in $A$ and by $H^{*}(X ; A)$ we denote the cohomology of a space $X$ with the twisted coefficients defined by $A$. Here we assume $\pi_{1}(X)=\pi$.

The Berstein-Schwarz class of a group $\pi$ is a certain cohomology class $\beta_{\pi} \in$ $H^{1}(\pi, I(\pi))$ where $I(\pi)$ is the augmentation ideal of the group ring $\mathbb{Z} \pi$ [1], [9], [17]. It is defined as the first obstruction to a section over $B \pi=K(\pi, 1)$ for the universal covering $p: E \pi \rightarrow B \pi$. Equivalently $\beta_{\pi}$ can be defined as follows. Let

$$
0 \rightarrow I(\pi) \rightarrow \mathbb{Z} \pi \stackrel{\epsilon}{\rightarrow} \mathbb{Z} \rightarrow 0
$$


be a short exact sequence of coefficients where $\epsilon$ is the augmentation homomorphism. Then $\beta_{\pi}=\delta(1)$ equals the image of the generator $1 \in H^{0}(\pi ; \mathbb{Z})=\mathbb{Z}$ under the connecting homomorphism $\delta: H^{0}(\pi ; \mathbb{Z}) \rightarrow H^{1}(\pi ; I(\pi))$ in the coefficient long exact sequence.

Theorem 9 (Universality). [9, Proposition 2.2], 17] For any $\pi$-module $L$ and any cohomology class $\alpha \in H^{k}(\pi, L)$ there is a homomorphism of $\pi$-modules $I(\pi)^{k} \rightarrow L$ such that the induced homomorphism for cohomology takes $\left(\beta_{\pi}\right)^{k} \in H^{k}\left(\pi ; I(\pi)^{k}\right)$ to $\alpha$ where $I(\pi)^{k}=I(\pi) \otimes \cdots \otimes I(\pi)$ and $\left(\beta_{\pi}\right)^{k}=\beta_{\pi} \smile \cdots \smile \beta_{\pi}$.

Corollary 10 ([17]). The class $\left(\beta_{\pi}\right)^{n}$ is the primary obstruction to a section of $p_{B \pi}^{n-1}: G_{B \pi}^{n} \rightarrow B \pi$.

Corollary 11. For any group $\pi$ its cohomological dimension can be expressed as follows:

$$
c d(\pi)=\max \left\{n \mid\left(\beta_{\pi}\right)^{n} \neq 0\right\} .
$$

3.3. Pasting sections. We recall that a map $p: E \rightarrow B$ satisfies the Homotopy Lifting Property for a pair $(X, A)$ if for any homotopy $H: X \times I \rightarrow B$ with a lift $H^{\prime}: A \times I \rightarrow E$ of the restriction $\left.H\right|_{A \times I}$ and a lift $H_{0}$ of $\left.H\right|_{X \times 0}$ which agrees with $H^{\prime}$, there is a lift $\bar{H}: X \times I \rightarrow E$ of $H$ which agrees with $H_{0}$ and $H^{\prime}$. We recall that a pair of spaces $(X, A)$ is called an NDR pair if $A$ is a deformation retract of a neighborhood in $X$. In particular, every CW complex pair is an NDR pair. It is well-known [20], Corollary 5.5.3 that any Hurewicz fibration $p: E \rightarrow B$ satisfies the Homotopy Lifting Property for NDR pairs $(X, A)$.

Lemma 12. Let $p: E \rightarrow B$ be a Hurewicz fibration over a $C W$ complex $B$ with $(n-1)$-connected fiber $F$ where $B=X \cup Y$ is presented as the union of subcomplexes with $n$-dimensional intersection $C=X \cap Y$, $\operatorname{dim} C=n$, such that $H^{n}(C ; \mathcal{F})=0$ for any local coefficients. Suppose that there are sections of $p$ over $X$ and $Y$. Then $p$ admits a section $s: B \rightarrow E$.

Proof. Let $s_{X}: X \rightarrow E$ and $s_{Y}: Y \rightarrow E$ be the sections. First we show that there is a fiberwise homotopy between the restrictions $s_{X}$ and $s_{Y}$ to $C$. We note that construction of such homotopy is a relative lifting problem

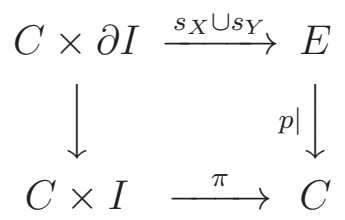

for the projection map $\pi$. Since the fiber is $(n-1)$-connected, a lift of $\pi$ exists on the $n$-skeleton $(C \times I)^{(n)}=\left(C^{(n-1)} \times I\right) \cup(C \times \partial I)$. The obstruction to extend it to the $(n+1)$-skeleton lives in the cohomology group $H^{n+1}(C \times I, C \times \partial I ; \mathcal{F})$ for local coefficients defined by $\pi_{n}(F)$. The exact sequence of pair together with the acyclicity of $C$ in dimensions $n$ and $n+1$ imply that $H^{n+1}(C \times I, C \times \partial I ; \mathcal{F})=0$. This proves the existence of a fiberwise homotopy $H: C \times I \rightarrow E$ between the sections $s_{X}$ and $s_{Y}$ restricted to $C$. In view of the Homotopy Lifting Property for NDR pairs the fiberwise homotopy $H: C \times I \rightarrow E$ of the restriction $\left.s_{X}\right|_{C}$ can be extended to a fiberwise homotopy $\bar{H}: X \times I \rightarrow E$ of $s_{X}$. Then the section $s_{X}^{\prime}: X \rightarrow E$ defined as $s_{X}^{\prime}(x)=H(x, 1)$ agrees with $s_{Y}$ on $C$. Hence the union $s_{X}^{\prime} \cup s_{Y}$ defines a section $s: X \cup Y \rightarrow E$. 


\section{Proof of Theorem 2}

Proof of Theorem 11. Corollary 11 and the cup-length lower bound for the LScategory imply that cat $G \geq c d(G)$. The dimension upper bound for the LS-category completes the proof for the groups with $c d(G)=\operatorname{gdim}(G)$. Now suppose that $c d(G)=$ 2 and $\operatorname{dim} B G=3$. Note that the Ganea-Schwarz fibration $G_{B G}^{2} \rightarrow B G$ has simply connected fiber. Thus, there is a section $s^{\prime}: B G^{(2)} \rightarrow G_{B G}^{2}$. The primary (and the only) obstruction to define a section $s: B G \rightarrow G_{B G}^{2}$ lives in the group $H^{3}(B G ; \mathcal{F})$ which is trivial in view of the equality $c d(G)=2$.

Proposition 13. For all groups $c d(G \times H) \geq c d(G)+1$.

Proof. We may assume that the groups have finite cohomological dimension. In particular, $H$ is torsion free. Hence it contains a copy of integers. Since the cohomological dimension of a subgroup does not exceed the cohomological dimension of a group [2], it follows that

$$
c d(G \times H) \geq c d(G \times \mathbb{Z}) \geq c d(G)+1
$$

Proof of Theorem 2. Since both $G$ and $H$ are not counterexamples to the EilenbergGanea conjecture, there are classifying spaces $B G$ and $B H$ with $\operatorname{dim} B G=c d(G)$ and $\operatorname{dim} B H=c d(H)$.

In view of Theorem 1 and Proposition 13 we obtain

$$
\operatorname{cat}(B G \times B H)=\operatorname{cat}(G \times H)=c d(G \times H) \geq \max \{c d(G), c d(H)\}+1 .
$$

Thus, by the Eilenberg-Ganea theorem

$$
\operatorname{cat}(B G \times B H) \geq \max \{\operatorname{dim}(B G), \operatorname{dim}(B H)\}+1 .
$$

By Theorem [6] we obtain

$$
\begin{gathered}
\mathrm{TC}(G * H)=\mathrm{TC}(B G \vee B H)=\max \{\mathrm{TC} G, \mathrm{TC} H, \operatorname{cat}(B G \times B H)\}= \\
\max \{\mathrm{TC} G, \mathrm{TC} H, \operatorname{cat}(B(G \times H))\}=\max \{\mathrm{TC} G, \mathrm{TC} H, c d(G \times H)\} .
\end{gathered}
$$

Problem 14. Suppose that $G$ is a counterexample to the Eilenberg-ganea conjecture, i.e. $\operatorname{cd}(G)=2$ and $\operatorname{gdim}(G)=3$. Does it follow that $\mathrm{TC}(G)=4$ ?

\section{Proof of Theorems 6, 7, 8}

Let $Y$ be a subspace of $X$. Then the inclusion $i: Y \rightarrow X$ gives rise to the map of fibrations $i_{\Delta}: \Delta_{Y}^{k} \rightarrow \Delta_{X}^{k}$. In particular, if $\Delta_{Y}^{k}$ admits a section over $Y \times Y$, then $\Delta_{X}^{k}$ also admits a section over $Y \times Y \subset X \times X$. Suppose, furthermore, that there is a retraction $r: X \rightarrow Y$. It gives rise to the map of fibrations $r_{\Delta}: \Delta_{X}^{k} \rightarrow \Delta_{Y}^{k}$. In particular, the existence of a section of $\Delta_{X}^{k}$ implies the existence of a section of the fibration $\Delta_{Y}^{k}$.

Similarly, given two topological spaces $X$ and $Y$, for each $k \geq 0$, there is a fibration $G_{X \times Y}^{k}$, called the $k$-th Ganea fibration, over $X \times Y$ such that cat $(X \times Y) \leq k$ if and only if $G_{X \times Y}^{k}$ admits a section. A point in $G_{X \times Y}^{k}$ over $(x, y) \in X \times Y$ is a formal sum $\sum t_{i}\left(g_{i}, h_{i}\right)$ where $g_{i}$ and $h_{i}$ are paths from respectively $x$ and $y$ to the distinguished point in $X$ and $Y$ respectively. 
Theorem [6 is a partial case of Theorem [7. So we prove the latter.

The lower bound for $\mathrm{TC}(X \vee Y)$. In this subsection we give an alternative proof of the fact that the topological complexity $\mathrm{TC}(X \vee Y)$ of the pointed sum of topological spaces $X$ and $Y$ is bounded below by TC $X$, TC $Y$ and cat $(X \times Y)$ (Theorem 3.6 [6]). We note that this lower bound works without any conditions.

Since both $X$ and $Y$ are retracts of $X \vee Y$, the existence of a section of $\Delta_{X \vee Y}^{k}$ implies the existence of sections of $\Delta_{X}^{k}$ and $\Delta_{Y}^{k}$. In other words, the topological complexity of $X \vee Y$ is bounded below by TC $X$ and TC $Y$.

To show that TC $(X \times Y)$ is also bounded by cat $(X \times Y)$, consider the map of fibrations

$$
p: \Delta_{X \vee Y}^{k} \mid X \times Y \longrightarrow G_{X \times Y}^{k}
$$

of the restriction of $\Delta_{X \vee Y}^{k}$ to $X \times Y \subset(X \vee Y) \times(X \vee Y)$ where $k=\mathrm{TC}(X \vee Y)$. The map $p$ is given by

$$
\sum t_{i} f_{i} \mapsto \sum t_{i}\left(p_{1} \circ f_{i}, p_{2} \circ \bar{f}_{i}\right),
$$

where $p_{i}$ is the projection of $X \vee Y$ to the $i$-th factor, and $\bar{f}_{i}$ is the path $f_{i}$ traversed in the opposite direction. Suppose that the fibration $\Delta_{X \vee Y}^{k}$ admits a section $s_{\vee}$. Then the fibration $G_{X \times Y}^{k}$ also admits a section $s_{G}$ defined as $s_{G}(x, y)=p \circ s_{\vee}(x, y)$. Thus, cat $(X \times Y) \leq k$.

The upper bound for $\mathrm{TC}(X \vee Y)$. In this subsection we show that $\mathrm{TC}(X \vee Y)$ is bounded above by the maximum of TC $X$, TC $Y$ and cat $(X \times Y)$. Let $k$ be the maximum of TC $X, \mathrm{TC} Y$ and cat $(X \times Y)$. In particular, the fibrations $\Delta_{X}^{k}, \Delta_{Y}^{k}$ and $G_{X \times Y}^{k}$ admit sections. We need to show that $\mathrm{TC}(X \vee Y) \leq k$, i.e., the fibration $\Delta_{X \vee Y}^{k}$ admits a section.

We assume that $X$ and $Y$ are $r$-connected CW-complexes of dimension $\leq d$ with TC $X$ or TC $Y$ at least $(d+1) /(r+1)$ with $r \geq 0$. Without loss of generality we may assume that TC $X \geq(d+1) /(r+1)$. We have shown that $k=\mathrm{TC}(X \vee Y) \geq \mathrm{TC} X$. Thus, $k \geq(d+1) /(r+1)$.

Note that if $Z$ is an $(r-1)$-connected space, then $*^{k+1} Z$ is $(k+(k+1) r-1)$ connected. Indeed, the join $*^{k+1} Z$ of spaces is homotopy equivalent to the reduced join of spaces, which, in its turn, is homeomorphic to the reduced suspension $\Sigma^{k}(Z \wedge$ $\cdots \wedge Z$ ), where the number of pointed factors is $k+1$. We may assume that besides the distinguished point $\Sigma^{k}(Z \wedge \cdots \wedge Z)$ has no cells in dimensions below $k+(k+1) r$. Therefore it is $(k+(k+1) r-1)$-connected. In particular, if $X$ and $Y$ are $r$-connected, then $\Omega(X \vee Y)$ is $(r-1)$-connected, and $*^{k+1} \Omega(X \vee Y)$ is $k+(k+1) r-1$-connected,

$$
k+(k+1) r-1=k r+k+r-1=k(r+1)+r-1 \geq(d+1)+r-1 \geq d .
$$

Thus, the fiber of $\Delta_{X \vee Y}^{k}$ is at least $d$-connected. We show that if $\Delta_{X \vee Y}^{k}$ admits sections over $X \times X, X \times Y, Y \times X$, and $Y \times X$, then it admits a section over $(X \vee Y)^{2}$. Let $A=(X \times X) \cup(Y \times Y)$ and $B=(X \times Y) \cup(Y \times X)$. Sections over $X \times X, X \times Y, Y \times X$, and $Y \times X$ can be taken such that they agree at $\left(x_{0}, x_{0}\right)$ where $x_{0}$ is the wedge point. Thus, there are sections over $A$ and $B$. Note that the intersection $A \cap B=X \vee X \vee Y \vee Y$ is $d$-dimensional. Since the fiber of the fibration is $d$-connected, Lemma 12 implies that that there is a section over $A \cup B=(X \vee Y)^{2}$.

In view of the retractions $X \vee Y \rightarrow X$ and $X \vee Y \rightarrow Y$ and the fact that $\Delta_{X}^{k}$ and $\Delta_{Y}^{k}$ have sections, the fibration $\Delta_{X \vee Y}^{k}$ admits sections over $X \times X$ and $Y \times Y$. Let 
us show that it also admits a section over $X \times Y$; the case of $Y \times X$ is similar. To this end, consider a map of fibrations

$$
q: G_{X \times Y}^{k} \longrightarrow \Delta_{X \vee Y}^{k} \mid X \times Y
$$

that takes a point $\sum t_{i}\left(g_{i}, h_{i}\right)$ in the fiber over $(x, y)$ to the point $\sum t_{i}\left(g_{i} \cdot \bar{h}_{i}\right)$ where - stands for taking the concatenation of two paths. Since $G_{X \times Y}^{k}$ admits a section $s$, the map $q$ of fibrations gives rise to the section $q \circ s$ of $\Delta_{X \vee Y}^{k}$ over $X \times Y$. This completes the proof of the upper bound for $\mathrm{TC}(X \vee Y)$.

Theorem 6 can be generalized to the following

Theorem 15. Let $d=\max \{\operatorname{dim} X, \operatorname{dim} Y\}$ for connected $C W$-complexes $X$ and $Y$. Suppose that $k=\max \{\mathrm{TC} X, \mathrm{TC} Y$, cat $(X \times Y)\} \geq d$ and $H^{k}(X ; \mathcal{F})=0=H^{k}(Y ; \mathcal{G})$ for all local coefficients. Then

$$
\mathrm{TC}(X \vee Y)=\max \{\mathrm{TC} X, \mathrm{TC} Y, \operatorname{cat}(X \times Y)\} .
$$

Proof. If $k \geq d+1$, the result follows from Theorem 6. We may assume that $k=d$. We need to check the inequality $\mathrm{TC}(X \vee Y) \leq k$. We use the above proof for $r=0$ to show that $\Delta_{X \vee Y}^{k}$ has $(d-1)$-connected fiber. Then $\Delta_{X \vee Y}^{k}$ admits a section over $(X \vee Y)^{2}=A \cup B$ by Lemma 12, since the intersection $A \cap B$ is $d$-dimensional and cohomologically acyclic in dimension $d$.

5.1. Proof of Theorem 8. Let $k=\max \{\mathrm{TC} X$, TC $Y$, cat $(X \times Y)\}$. We show that the fibration $\Delta_{X \cup Y}^{k}$ admits a section. Since TC $X$, TC $Y \leq k$, it admits a section over $X \times X$ and $Y \times Y$. Choosing a common base point $c_{0}$ for $X$ and $Y$ allows us to embed the base point path space $P_{0}(X \times Y)$ to the path space $P(X \cup Y)$ by taking a path $(f, g)$ in $X \times Y$ issued from the base point $\left(c_{0}, c_{0}\right)$ to the path $\bar{f} g$. This defines an embedding of the Ganea-Schwarz fibration $G_{X \times Y}^{k}$ in the fibration $\Delta_{X \cup Y}^{k}$. The inequality cat $(X \times Y) \leq k$ implies that $G_{X \times Y}^{k}$ has a section. Therefore, $\Delta_{X \cup Y}^{k}$ has a section over $X \times Y$. Similarly, it has a section over $Y \times X$.

The rest of the argument is similar to those of Theorem 3 and 4 . We consider the sets $A=X \times X \cup Y \times Y$ and $B=X \times Y \cup Y \times X$ and argue that both sets admit sections of $\Delta_{X \cup Y}^{k}$. For example, in the case of $A$ the two sections over $X \times X$ and $Y \times Y$ can only disagree over $C \times C$ which has dimension $\leq d$ where $C=X \cap Y$. Since the fibration $\Delta_{X \cup Y}^{k}$ has $d$-connected fiber those two sections can be joined over $C \times C$ by a fiberwise homotopy. This implies the existence of a section over $A$. The argument in the case of $B$ is similar. Next we note that $A \cap B=X \times C \cup Y \times C \cup C \times X \cup C \times Y$ is $d$-dimensional. The same argument implies that there is a continuous section over $A \cup B=(X \cup Y) \times(X \cup Y)$.

\section{Amalgamated product}

Theorem 16. If all groups $A, B$, and $C$ are not counterexamples to the EilenbergGanea conjecture, then

$$
\mathrm{TC}\left(A *_{C} B\right) \leq \max \{\mathrm{TC} A, \mathrm{TC} B, c d(A \times B), c d(A *(C \times \mathbb{Z}) * B)+c d(C)+1\} .
$$

Proof. Let classifying spaces $B A, B B$, and $B C$ for groups $A, B$, and $C$ be such that $c d(A)=\operatorname{dim} B A, c d(B)=\operatorname{dim} B B$, and $c d(C)=\operatorname{dim} B C$. Here we used that the groups are not potential counterexamples to the Eilenberg-Ganea conjecture. For the group $G=A *_{C} B$ we consider its classifying space $B G$ of the form of the double 
mapping cylinder for the maps $f: B C \rightarrow B A$ and $g: B C \rightarrow B B$ induced by the amalgamation homomorphisms $C \rightarrow A$ and $C \rightarrow B$. Thus, $B G=X \cup Y$ with $X \cap Y=B C, X$ is homotopy equivalent to $B A$ with $\operatorname{dim} X=\max \{c d(A), c d(C)+1\}$, and $Y$ is homotopy equivalent to $B B$ with $\operatorname{dim} Y=\max \{c d(B), c d(C)+1\}$. Let

$$
\begin{gathered}
d=\max \{\operatorname{dim}(X \times B C), \operatorname{dim}(Y \times B C)\}=\max \{\operatorname{dim} X, \operatorname{dim} Y\}+c d(C)= \\
\max \{c d(A), c d(C)+1, c d(B)\}+c d(C)=c d(A *(C \times \mathbb{Z}) * B)+c d(C) .
\end{gathered}
$$

If

$$
\max \{\mathrm{TC} A, \mathrm{TC} B, \operatorname{cat}(A \times B)\} \geq d+1,
$$

then the result follows from Theorem 8 . Suppose that

$$
\max \{\mathrm{TC} A, \mathrm{TC} B, \operatorname{cat}(A \times B)\} \leq d .
$$

We show that $\Delta_{B G}^{d+1}$ admits a section. Since TC $A=\mathrm{TC} X \leq d+1$ and TC $B=$ TC $Y \leq d+1$, there are sections over $X \times X$ and $Y \times Y$. Since cat $(X \times Y) \leq d+1$ there are section over $X \times Y$ and $Y \times X$. Since $(X \times X) \cap(Y \times Y)=B C \times B C$

with $\operatorname{dim}(B C \times B C)=2 c d(C) \leq d$ and the fiber of $\Delta_{B G}^{d+1}$ is $d$-connected, sections over $X \times X$ and $Y \times Y$ can be adjusted over $B C \times B C$ to have a continuous section over $U=(X \times X) \cup(Y \times Y)$. Similarly, we can arrange a continuous section over $V=(X \times Y) \cup(Y \times X)$. Note that

$$
U \cap V=(X \times B C) \cup(Y \times B C) \cup(B C \times X) \cup(B C \times Y) .
$$

Thus $\operatorname{dim}(U \cap V)=d$. Therefore, we can arrange a continuous section over $U \cup V=$ $B G \times B G$.

\section{REFERENCES}

[1] I. Berstein, On the Lusternik-Schnirelmann category of Grassmannians. Math. Proc. Cambridge Philos. Soc. 79 (1976), no. 1, 129-134.

[2] K. Brown, Cohomology of groups, Springer, New York-Heidelberg-Berlin, 1982.

[3] G. Bredon, Sheaf Theory. Graduate Text in Mathematics, 170, Springer, New YorkHeidelberg-Berlin, 1997.

[4] D. Cohen, L. Vandembroucq, Topological complexity of the Klein bottle, J. Appl. and Comput. Topology (2017) https://doi.org/10.1007/s41468-017-0002-0.

[5] O. Cornea; G. Lupton; J. Oprea; D. Tanre, Lusternik-Schnirelmann Category. Mathematical Surveys and Monographs, 103. American Mathematical Society, Providence, RI, 2003.

[6] A. Dranishnikov, Topological complexity of wedges and covering maps, Proc. AMS 142 (2014), 4365-4376.

[7] A. Dranishnikov, On the virtual cohomological dimensions of Coxeter groups. Proc. Amer. Math. Soc. 125 (1997), no. 7, 1885-1891.

[8] A. Dranishnikov, The topological complexity and homotopy cofiber of the diagonal map of non-orientable surfaces, Proc. Amer. Math. Soc. 144 (2016), no 11, 4999-5014.

[9] A. Dranishnikov, Yu. Rudyak, On the Berstein-Svarc theorem in dimension 2. Math. Proc. Cambridge Philos. Soc. 146 (2009), no. 2, 407-413.

[10] S. Eilenberg, T. Ganea, On the Lusternik-Schnirelmann Category of Abstract Groups, Annals of Mathematics, 65, (1957), 517-518.

[11] M. Farber, Invitation to topological robotics. Zurich Lectures in Advanced Mathematics. European Mathematical Society (EMS), Zurich, 2008.

[12] M. Farber, Topology of robot motion planning. Morse theoretic methods in nonlinear analysis and in symplectic topology, 185-230, NATO Sci. Ser. II Math. Phys. Chem., 217, Springer, Dordrecht, 2006.

[13] M. Farber, S Mescher, On the topological complexity of aspherical spaces, Preprint arXiv:1708.06732v2 [math.AT]. 
[14] M. Grant, Topological complexity, fibrations and symmetry, Topol. Appl. 159 (2012), no $1,88-97$.

[15] N. Iwase, M. Sakai, Topological complexity is a fibrewise L-S category. Topology Appl. 157 (2010), no. 1, 10-21.

[16] Yu. Rudyak, On topological complexity of Eilenberg-MacLane spaces. Topology Proc. 48 (2016), 65-67.

[17] A. Schwarz, The genus of a fibered space. Trudy Moscov. Mat. Obsc. 10, 11 (1961 and 1962), 217-272, 99-126.

[18] J. Stallings, On torsion-free groups with infinitely many ends, Ann. of Math. 88 (1968), 312-334.

[19] R. Swan, Groups of cohomological dimension one, J. Algebra 12 (1969), 585-610.

[20] Tammo tom Dieck, Algebraic Topology. European Mathematical Society, 2008.

A. Dranishnikov, Department of Mathematics, University of Florida, 358 Little Hall, Gainesville, FL 32611-8105, USA

E-mail address: dranish@math.ufl.edu

R. Sadykov, Mathematics Department, Kansas State University, 138 Cardwell Hal, L Manhattan, KS 66506

E-mail address: sadykov@math.ksu.edu 